\title{
The Unibo energy saving and climate change approach
}

\author{
Mirko Degli Esposti ${ }^{1^{*}}$, Olivia Bernardi ${ }^{2}$, and Luciana Sacchetti ${ }^{3}$ \\ ${ }^{1}$ Alma Mater Studiorum - Università di Bologna, Deputy Rector, Professor of Mathematical Physics, Italy \\ ${ }^{2}$ Alma Mater Studiorum - Università di Bologna, Sustainability Office, Italy \\ ${ }^{3}$ Alma Mater Studiorum - Università di Bologna, Research and Third Mission Division, Italy
}

\begin{abstract}
For the university of Bologna, the core of sustainability combines both environmental and social dimensions: enhancement of the territory, ensuring its protection and the renewal of its natural resources and the ability of the parties involved, encouraged by the concerted efforts of the various institutions, to work together effectively. The commitment of the university is to reduce the impact on the environment deriving from its activities and to encourage the community to assume responsible and environmentally respectful behaviours. Since 2016, the university integrates its Strategic Plan with a rereading of the $17 \mathrm{UN}$ SDGs and reports the direct and indirect impact produced in the dimensions of teaching, research and third mission.
\end{abstract}

\section{Introduction}

The Alma Mater Studiorum - Università di Bologna founded in 1088 and recognized as the oldest university in the Western world, today is one of the most important institutions of higher education throughout Europe with more than 84,000 registered students, 2850 academics, 3100 administrative employees and over 3700 young scientists.

Managing more than 1,000,000 sq. m. of modern and historical buildings of the university citadels located throughout the region and beyond, the University of Bologna plays a decisive role within the urban, regional and national dynamics.

For the Alma Mater it is natural to be a promoter of the principles of sustainability such as the enhancement and protection of the territory, the improvement of community welfare, the economic development, the social equity and the capacity of the subjects involved to work together effectively for the common good.

To achieve objectives in terms of sustainability the university of Bologna takes a project-based approach towards pragmatic results. That involves adoption of innovative methodologies for the measurement of results and control of the various operational processes, defined in the 2016-2021 Sustainable Multicampus Project [1].

\section{The university strategic plan 2016- 2018 and the UN SDG's}

Since 2016 the university's governance foresaw to develop the university Strategic Plan [2] following the 2030 Agenda and the sustainable development goals of the United Nations (SDG's) for tackling the challenges posed by contemporary reality and in particular for a sustainable development.

This innovative approach has led to re-read the university's activities to allow the insertion in everyday life of the values and the communicative and inspiring power of the SDGs, with a real impact on the university's community and its stakeholders.

Unibo has integrated its Strategic Plan with a full rereading of the 17 basic sustainable development goals. Every basic target in the Strategic Plan is associated with one or more of the sustainable development goals (Fig.1).

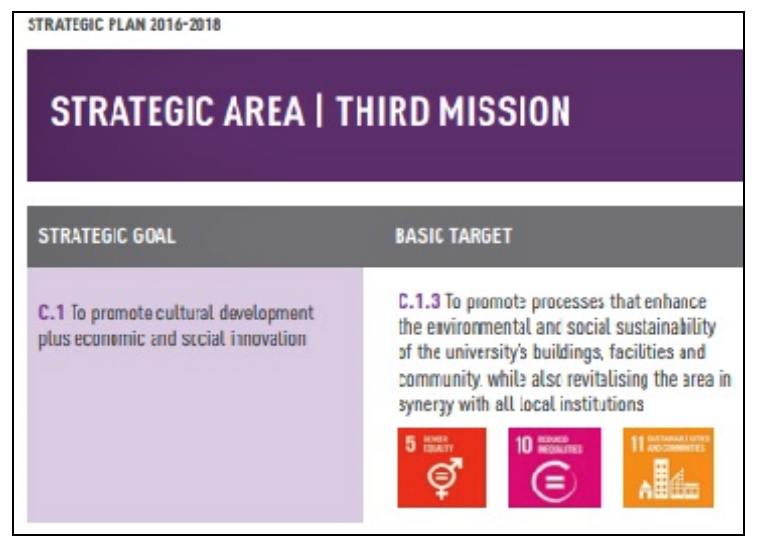

Fig. 1. the strategic Plan's basic targets and the related UN sustainable goals.

To the area of the Third Mission is associated the strategic goal "to promote cultural development plus economic and social innovation". In this framework, the basic target C.1.3 recites as "to promote processes that enhance the environmental and social sustainability of the university's buildings, facilities and community, while also revitalizing the area in synergy with all local

\footnotetext{
Corresponding author: mirko.degliesposti@unibo.it
} 
institutions". The actions planned to reach that target are aligned with three SDGs: 5 Gender's equality, 10 reduce inequality, 11 sustainable cities and communities.

\section{The report on U.N. sustainable development goals 2016}

Consistent with the perspectives adopted in the Strategic Plan 2016-2018, the University has an innovative way to report on the contributions generated by its institutional activities, aimed at the achievement of the 17 SDGs. The report [3] presents 17 sections, each one dedicated to a UN goal, reporting the impact generated by the university of Bologna through its diverse activities. Each goal is reported in relation to the four academic dimensions of training, research, third mission and the institution.

The report and its metrics were formulated by the Scientific and Technical Committee for Social Reporting at the University of Bologna. Data refers to 2016 as well as the Academic Year 2016/17 for training data. The original metrics match and integrate other official document of the Alma Mater, a methodological note on page 62-65, gives a full description of each metric cited in table 1.

Table 1. The reporting metrics

\begin{tabular}{|l|l|l|l|}
\hline $\begin{array}{c}\text { Publications } \\
\text { in Scopus }\end{array}$ & $\begin{array}{c}\text { "Cited } \\
\text { by" in } \\
\text { Scopus }\end{array}$ & $\begin{array}{c}\text { International } \\
\text { benchmarking }\end{array}$ & $\begin{array}{c}\text { National } \\
\text { benchmarking }\end{array}$ \\
\hline Course Unit & Students & $\begin{array}{l}\text { Collaborations, } \\
\text { teaching } \\
\text { mobility }\end{array}$ & $\begin{array}{l}\text { FP7, H2020 } \\
\text { research } \\
\text { projects }\end{array}$ \\
\hline $\begin{array}{l}\text { Cooperation } \\
\text { \& social } \\
\text { engagement } \\
\text { research } \\
\text { projects }\end{array}$ & $\begin{array}{l}\text { Patents } \\
\text { including } \\
\text { plant } \\
\text { varieties) }\end{array}$ & & \\
\hline
\end{tabular}

\section{Take urgent action to combat climate change and its impact - SDG 13}

The impact of the university on the sustainable development goal 13 (Fig. 2) is described in the report from three different perspectives: teaching, research and from the viewpoint of the "institution". Evidences for the teaching engagement on climate change are the 54 course units and the 4,794 students who could choose the course unit.

From the research perspective, 6 publications during the period 2007-2016 could be fully identified in Scopus as related to climate change and its impact, generating 164 citations. The bibliometric outputs are responsible for ranking Bologna below the international benchmark (100) for publication per capita (Unibo index number 30), but above the national benchmark which consider the universities of Padua, Rome La Sapienza, Milano Statale (Unibo index number 130). More relevant is the impact of the transportation actions taken by the
University as an institution, such as the use of public transport on favourable terms, an electric fleet management and the promotion of bicycle. As certified by GreenMetric 2016 on 2015 data, the Alma Mater CO2 emission over the last 12 months is about 512 Tons.

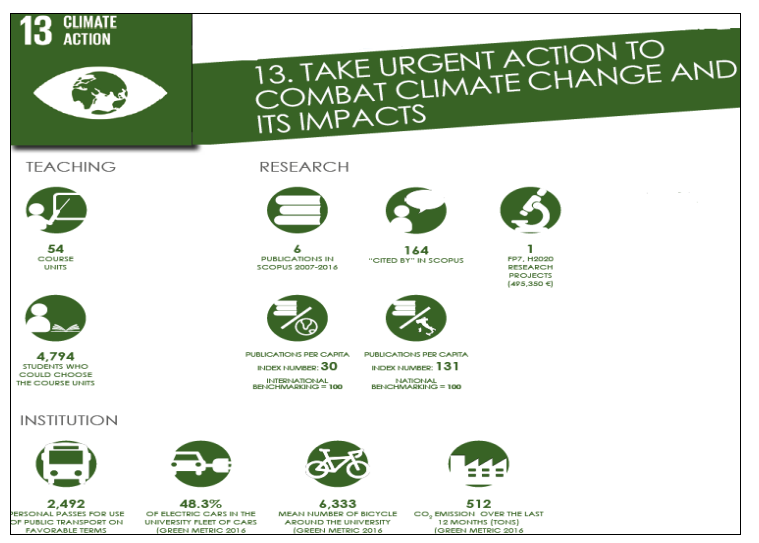

Fig. 2. the climate action at the University of Bologna.

\section{Actions for sustainability}

\subsection{Energy}

In order to reduce energy consumption, the University of Bologna strives to make technological and architectonic enhancements and encourages the University population to use energy more rationally. The measures identified seek to respond to the many requirements stemming from the constant improvement of buildings and to facilitate the rationalization of their differing energy needs.

The commitment of the University in the area of energy saving is designed to:

o Improve awareness, as well as the management and control of building energy consumption, by adopting measures to obtain and manage energy data in real time, and to control heating and air-conditioning installations on a remotely.

o Improve architectonic efficiency, by adopting measures that increase the energy efficiency of buildings and their heat insulation.

o Improve the efficiency of systems by implementing technological upgrades and optimizing the configuration of the heating and cooling systems installed.

o Encourage and spread the rational use of energy, as a key element in the reduction of waste.

The principal actions undertaken are:

Renewable energy - The university harnesses the renewable energy sourced from photovoltaic installations: Unibo has more than 8,500 sq. m. of photovoltaic surface in its buildings.

Remote monitoring and energy management - The University of Bologna monitors the consumption of utilities on a remote basis. The GECO project (Energy Management and Remote Control) seeks the maximum energy saving of buildings, by controlling energy flows and environmental parameters. 
Smart building implementation - The university has applied a program of smart building implementation, providing several energy efficient appliances and tools. In almost all buildings is installed a network technology, in order to control facility functions, such as thermal power plant management system, internal volumetric sensor installed for physical security, remote control of an external lights system, automatic light and Fire Alarm sensor system, dashboard of the integrated energy management system.

Replacement of lighting - The University of Bologna guarantees the constant upgrade of internal and external lighting. Moreover, we are experimenting innovative remote management and remote-control systems for lighting bodies and progressively subsitute obsolete lighting fixtures with high-energy consumption with innovative systems.

\subsection{Climate change}

As a contribution to the protection of the environment, the university promotes the enhanced use of green areas, a more responsible use of water and waste, to help improve the quality of life in University areas. Via the rational use of natural resources in its buildings, the university pursues the objective of harmonizing the essential trade-off between built-up areas and the surrounding environment.

In addition to encouraging students and staff to dedicate greater attention to environmental matters, the approach adopted seeks to highlight the value of our territory and landscapes. Moreover, in order to reduce atmospheric emissions, the University of Bologna promotes a low impact mobility both when at work and when travelling between home and work/place of study.

Our commitment to mobility is designed to:

o Encourage public transport by adopting measures to reduce the number of private vehicles used.

o Lower atmospheric emissions by promoting the use of cycle paths and other forms of low-impact transportation.

o Offer innovative and sustainable mobility services aimed at the shared use of vehicles.

The actions taken to implement our commitment are the following:

Reduce and recycling waste - we manage the various types of waste produced by its structures with a view to proper separation, safety and recycling. Unibo has established formal policy to reduce the use of paper and plastic and separate waste for recycling. The university has provided suitable containers (about 10.000) for paper, plastic, glass and aluminium, batteries and toner cartridge, located in all the buildings. Moreover, the toxic waste are completely contained, inventoried and handled.
Green roof - The university installed approximately 1,000 sq. $\mathrm{m}$. of green roofs and hanging gardens on the horizontal roof plans of the university buildings.

Green areas - The university increases the amount of green by transforming the use of existing areas, renovating green ones abandoned and developing the use of greenery in urban settings.

Water - The university strives to make best use of the available water resources, in order to contain the amount of water wasted not only with water efficient appliances but also with automatic irrigation systems.

Public transport - The university encourages public transport enabling students and personnel to purchase passes for the use of public transport on favourable terms agreed with the transport companies and municipal administrations.

Electric cars and car sharing - The University of Bologna has rationalized and renewed its fleet of cars, which are all-electric and used in car sharing mode.

Bicycle - we encourage the use of bicycles by providing each building with bicycle parking and bicycle paths. Moreover, in order to disseminate a culture of sustainable mobility the university has signed an agreement with the Bologna bike station that enables the university community to use its various services on advantageous terms: bike rental, secure parking and repair/self-repair facilities.

\section{Concluding remarks}

The Alma Mater Studiorum is committed to spreading a real culture of sustainability both as National coordinator of the UI GreenMetric Network and in collaboration with the RUS - University Network for Sustainable Development [4], which principal goal is to increase the environmental, ethical, social and economic impact of the actions implemented by the Italian universities.

\section{References}

1. 2016-2021 Sustainable Multicampus Project available online at http://www.sostenibilita.unibo.it/unibosostenibile/progetto-multicampus-sostenibile

2. University Strategic Plan 2016-2018 available online at http://www.unibo.it/en/university/ whowe-are/strategic-plan

3. Report on U.N. Sustainable Development Goals 2016, page 67 available online at http://www.unibo.it/it/ateneo/chi-siamo/reportingon-united-nations-sustainable-development-goals

4. RUS - University Network for Sustainable Development available online at https://sites.google.com/unive.it/rus/ 